\title{
Comportamiento corneal inmediato tras facoemulsificación, por medio de tomografía de coherencia óptica de dominio espectral
}

\section{Immediate corneal behavior after phacoemulsification, by means of spectral-domain optical coherence tomography}

\author{
Luis R. Alvarez-Fernández ${ }^{*}$, Oscar Baca-Lozada ${ }^{1}$, Héctor Pérez-Cano², Regina Velasco-Ramos ${ }^{1}$, \\ Elisa D. Alegría-Gómez y Oscar Fernández-Vizcaya ${ }^{1}$
}

${ }^{1}$ Departamento de Córnea y Cirugía Refractiva; ${ }^{2}$ Departamento de Investigación. Fundación Hospital Nuestra Señora de la Luz Institución de Asistencia Privada, Ciudad de México, México

\begin{abstract}
Resumen
Objetivo: Describir los cambios corneales en las 24 horas posteriores a la facoemulsificación, por medio de tomografía de coherencia óptica de dominio espectral (OCT-SD). Método: Estudio prospectivo, observacional, descriptivo, transversal y comparativo. Se utilizaron imágenes tomadas por OCT-SD en ojos sin antecedentes de cirugía intraocular y corneal, para determinar un promedio de grosor, densidad, reflectividad y características normales de cada capa corneal, en tres grupos etarios de 50-59 años, 60-69 años y 70-79 años. Se describieron las características de cada córnea en relación con el tiempo efectivo de facoemulsificación (EPT). Resultados: Se analizaron 180 ojos, en donde el grosor etarios total en los tres grupos se encontró una $p<0.0001$ comparando las córneas de los ojos operados y no operados, en donde se obtuvo un aumento del 13\% entre el grupo 1 y 2, y un aumento del 1\% entre el grupo 2 y 3. La densidad comparada por grupos mostró $p=0.0028$, $p=0.0011$ y $p<0.0001$, con una disminución de $-2.01 \%$ en el grupo 1, -4.13\% en el grupo 2 y $-5.07 \%$ en el grupo 3. A partir de un EPT > 8 segundos se encontró una disminución en la reflectividad del estroma, la membrana de Descemet y el endotelio. Conclusiones: El aumento del grosor corneal es inversamente proporcional a la densidad corneal, la reflectividad corneal presenta cambios significativos conforme aumenta el EPT y la cirugía de facoemulsificación produce múltiples cambios corneales observables por OCT-SD, a pesar de que en la lámpara de hendidura en ocasiones no se perciban.
\end{abstract}

Palabras clave: Facoemulsificación. OCT-SD. Grosor. Densidad. Reflectividad. EPT.

\begin{abstract}
Objective: To describe the corneal changes in the first 24 hours after phacoemulsification, by means of spectral-domain optical coherence tomography (SD-OCT). Method: Prospective, observational, descriptive, cross-sectional and comparative study. Images taken by SD-OCT in eyes without a history of intraocular and corneal surgery were used to determine an average of thickness, density, reflectivity and normal characteristics of each corneal layer, in three age groups of 50-59 years, 60-69 years and 70-79 years. The characteristics of each cornea were described in relation to the effective phacoemulsification time (EPT). Results: We analyzed 180 eyes, finding a $p<0.0001$ for the total thickness in the 3 groups when comparing the corneas of the operated and non-operated eyes, where an increase of $13 \%$ was obtained between groups 1 and 2 , and an increase of $1 \%$ between groups 2 and 3. The density compared by groups showed $p=0.0028, p=0.0011$ and $p<0.0001$,
\end{abstract}

Correspondencia:

*Luis R. Alvarez-Fernández

Ezequiel Montes 135

Col. Tabacalera, Alcaldía Cuauhtémoc

Fecha de recepción: 08-02-2021

Fecha de aceptación: 04-08-2021

E-mail: iraf_raise@ hotmail.com

DOI: 10.24875/RMO.M21000200
Disponible en internet: 05-11-2021 Rev Mex Oftalmol. 2021;95(6):259-266

www.rmo.com.mx 0187-4519/@ 2021 Sociedad Mexicana de Oftalmología. Publicado por Permanyer. Este es un artículo open access bajo la licencia CC BY-NC-ND (http://creativecommons.org/licenses/by-nc-nd/4.0/). 
with a decrease of $-2.01 \%$ in group 1, $-4.13 \%$ in group 2 and $-5.07 \%$ in group 3 . From an EPT $>8$ seconds, a decrease in the reflectivity of the stroma, Descemet's membrane and endothelium was found. Conclusions: The increase in corneal thickness is inversely proportional to corneal density; corneal reflectivity shows significant changes as EPT increases and phacoemulsification surgery produces multiple corneal changes observable by SD-OCT, despite the fact that they are sometimes not perceived by slit-lamp examination.

Keywords: Phacoemulsification. SD-OCT. Thickness. Density. Reflectivity. EPT.

\section{Introducción}

La evaluación de la córnea es una parte importante del examen oftalmológico. Existen varias maneras de realizarla, como la visualización por medio de la lámpara de hendidura, la biomicroscopía con ultrasonido o la utilización de la tomografía de coherencia óptica de dominio espectral (OCT-SD). Esta última es una técnica que no utiliza el contacto corneal y provee una imagen de alta resolución que es utilizada en varias situaciones clínicas ${ }^{1}$. La OCT-SD se basa en la interferometría de baja coherencia, evaluando el retraso y la intensidad de la luz infrarroja retrodispersada utilizando un diodo que es superluminiscente. La longitud de onda es de aproximadamente $1310 \mathrm{~nm}$; esto mide los dos haces de luz comparando la reflectividad en el tejido, que se conoce como espejo de referencia. Estas rutas se envían a una computadora, realizando múltiples exploraciones longitudinales en una serie de ubicaciones laterales para formar una imagen bidimensional ${ }^{2}$.

La medición del grosor corneal es útil en el diagnóstico de ciertas patologías corneales, enfatizando su uso en las alteraciones que se producen tras una facoemulsificación. Al ser un estudio de alta resolución, puede ayudar a identificar prematuramente alteraciones anatómicas, como el edema estromal ${ }^{3}$. En la actualidad, las incisiones corneales claras son las más realizadas durante la facoemulsificación, ya que una propiedad importante y útil es su capacidad para cerrarse sin sutura, aunque esto ha aumentado el riesgo de endoftalmitis, por lo que se ha utilizado la OCT para evaluar cómo la hidratación estromal afecta la arquitectura de estas incisiones en el posoperatorio inmediato ${ }^{4}$. Por medio de OCT-SD se han identificado los grosores de las diferentes capas de una córnea sana en su porción central: el epitelio mide aproximadamente $50 \mu \mathrm{m}$, la membrana de Bowman $12 \mu \mathrm{m}$, el estroma $500 \mu \mathrm{m}$, la membrana de Descemet 4-10 $\mu \mathrm{m}$ y el endotelio $5 \mu \mathrm{m}^{5}$.

La transmisión de la luz a través de la córnea humana es gracias a la disposición de las fibras de colágeno, y la regulación de la deshidratación estromal es mantenida en el endotelio por una serie de bombas moleculares, moduladores químicos y propiedades de unión celular ${ }^{6}$. Las alteraciones corneales secundarias a la facoemulsificación se pueden presentar como resultado de una falla de la bomba endotelial posterior a la cirugía, debido a una lesión mecánica o química, por infección o por compromiso endotelial preexistente. Existen factores de riesgo asociados que condicionan desórdenes y pérdida de células endoteliales, desde factores prequirúrgicos, como distrofias endoteliales corneales, glaucoma, uveítis, síndrome de pseudoexfoliación y trauma corneal, hasta factores intraquirúrgicos, como la experiencia del cirujano, la incisión, la irrigación de soluciones, el tiempo quirúrgico y complicaciones vítreas y posquirúrgicas, como vítreo en cámara anterior, contacto endotelial del lente intraocular, dehiscencia de la herida, síndrome tóxico del segmento anterior y desprendimiento de la membrana de Descemet ${ }^{7,8}$.

El desprendimiento de la membrana de Descemet se puede detectar con la lámpara de hendidura si el edema corneal no es grave, aunque el estudio ideal es la $\mathrm{OCT}^{9,10}$. Las anormalidades resultantes en las acuaporinas, posterior a la irrigación de soluciones o los fármacos utilizados intraquirúrgicos, pueden producir edema corneal pseudofáquico. Se ha visto que la facoemulsificación produce daño a las células endoteliales de la córnea por la generación de radicales libres, como glutatión reducido, calcio, adenosina, hialuronato de sodio, superóxido dismutasa y ácido ascórbico. Las células endoteliales presentan uniones estrechas y discontinuas que son dependientes del calcio, por lo que el uso de soluciones de irrigación sin calcio puede reducir la función de barrera que conduce al edema corneal ${ }^{11}$.

El edema corneal se divide conforme a su temporalidad: de inicio inmediato, que abarca las primeras 24 horas y se caracteriza por trauma endotelial con falla celular funcional, que se asocia a excesiva energía utilizada durante la cirugía, larga duración, elevación de la presión intraocular y desprendimiento de la membrana de Descemet, y de inicio tardío, en donde el 
edema se manifiesta tras un periodo de claridad corneal que se caracteriza por pérdida progresiva de células endoteliales, como la queratopatía bullosa afáquica y pseudofáquica ${ }^{12}$. La elevación de la presión intraocular es indispensable manejarla con hipotensiores oculares como prevención de daño a las células endoteliales; los inhibidores de las prostaglandinas, los inhibidores de la anhidrasa carbónica y los esteroides deben ser utilizados con cautela en caso de edema corneal $^{13}$. Las opciones quirúrgicas dependen del inicio del edema corneal; si es inmediato, como en el desprendimiento de la membrana de Descemet, se utiliza gas o aire intracameral, y si es de inicio tardío, en casos de daño endotelial irreversible, se utiliza la queratoplastia penetrante 0 el trasplante lamelar posterior $^{14}$. Los desprendimientos de la membrana de Descemet tras la facoemulsificación se han clasificado en tres tipos: simple cuando solo se encuentra en la incisión, simétrico cuando se encuentra en la incisión y el limbo contralateral, y completo cuando abarca también la parte central ${ }^{15}$.

Actualmente existen varios tomógrafos de coherencia óptica de segmento anterior; por ejemplo, el OCT-SD Heidelberg Spectralis ${ }^{\circledR}$, que es un sistema de interferometría de baja coherencia con una alta resolución en la imagen ${ }^{16,17}$ y que ofrece la opción de añadir lentes positivas y negativas para compensar la ametropía del paciente, además de contar con una excelente resolución en la medición del grosor corneal y la ejemplificación de sus características, proporcionando mayor detalle, por lo que sería considerado como una «biopsia óptica»18.

El objetivo del presente estudio es describir los cambios corneales en las 24 horas posteriores a una facoemulsificación, por medio de OCT-SD.

\section{Método}

Estudio prospectivo, observacional, descriptivo, transversal y comparativo de las características corneales tras la facoemulsificación por medio de OCT-SD, en el año 2019. Se seleccionaron 90 pacientes de 50 a 79 años divididos en tres grupos: 50-59 años, 60-69 años y 70-79 años. A los grupos de ojos operados se les asignaron los números 1, 2 y 3 , y a los grupos de ojos no operados las letras A, B y C. Los pacientes fueron operados con un equipo Stellaris PC Baush \& Lomb $^{\circledR}$, documentando en la nota posquirúrgica el tiempo efectivo de facoemulsificación y que el ojo contralateral nunca haya tenido cirugía intraocular y tampoco patología corneal que afecte los $3 \mathrm{~mm}$ centrales.
Se realizó OCT-SD con el sistema Heidelberg Spectralis ${ }^{\circledR}$ en ambos ojos con zoom de $8.3 \mathrm{~mm}$, obteniendo una imagen con una calidad $>20 \mathrm{y}<35$, una sola toma en el posoperatorio inmediato en el transcurso de las primeras 24 horas (90 ojos operados) y una sola toma en los ojos contralaterales (90 ojos sanos). Se dividieron y analizaron grosores, densidades, reflectividades y características por grupo etario, y se obtuvo el valor del coeficiente de correlación con el tiempo efectivo de facoemulsificación.

Se obtuvo la densidad de las capas corneales por medio del programa Image $\mathrm{J}$ del National Institute of Health, calibrando la imagen a $200 \mu \mathrm{m}$, que es el valor estandarizado, midiendo la densidad en escala de grises de 256 píxeles 8-bit. Los datos obtenidos se ingresaron en una tabla del software Microsoft Excel con ficha de identificación de los pacientes. Se analizaron las características de las imágenes de OCT-SD de las capas corneales, los grosores, las reflectividades y las densidades, tanto en los ojos operados como en los no operados, en los $3 \mathrm{~mm}$ centrales fuera del artefacto.

Se realizaron gráficas para mostrar los resultados obtenidos comparando por grupo etario las características observadas. Para el análisis estadístico se ingresaron los resultados al programa GraphPad Prism V5.0, se realizó la prueba de Kolmogórov-Smirnov y al obtener resultados no paramétricos se realizó la prueba $\mathrm{U}$ de Mann-Whitney. En la correlación del EPT con la densidad y el grosor se realizó la prueba rho de Spearman. Se consideró estadísticamente significativo un valor de $p<0.05$.

\section{Resultados}

En el grosor corneal se encontró una p estadísticamente significativa en los tres grupos etarios ( $p<$ 0.0001) (Tabla 1), esto comparando los grupos 1, 2 y 3 con los grupos A, B y C, en los que se observa cómo el aumento del grosor total depende básicamente del aumento del grosor estromal con una prueba de relación de 0.5963 ( $p<0.0001)$ (Fig. 1). Sin embargo, también se encontró que la diferencia del grosor corneal de los pacientes operados entre el grupo 1 y el grupo 2 era del $13 \%$, pero la diferencia entre los grupos 2 y 3 es $<1 \%$, lo que indica que la edad es un factor protector en el grupo 1, en comparación con los grupos 2 y 3 ( $p<0.0001)$ (Fig. 2).

En la densidad corneal también se encontró significancia estadística en los tres grupos etarios: grupos 1 y $A(p=0.0028)$, grupos 2 y $B(p=0.0011)$, grupos 3 y $C(p<0.0001)$; esto comparando los grupos por edad 
Tabla 1. Grosor corneal. Los grupos 1, 2 y 3 corresponden a los ojos operados de facoemulsificación, y los grupos A, B y C corresponden a los ojos contralaterales no operados. Se presenta el valor promedio de grosores en micrómetros, y los valores que aparecen entre paréntesis corresponden a los máximos y mínimos

\begin{tabular}{|c|c|c|c|c|c|c|}
\hline \multirow[b]{2}{*}{ Grupos } & \multicolumn{2}{|c|}{ 50-59 años (n = 60) } & \multicolumn{2}{|c|}{ 60-69 años (n = 60) } & \multicolumn{2}{|c|}{ 70-79 años (n = 60) } \\
\hline & $1(n=30)$ & $A(n=30)$ & $2(\mathrm{n}=30)$ & $B(n=30)$ & $3(n=30)$ & $C(n=30)$ \\
\hline Epitelio $(\mu \mathrm{m})$ & (45-66) 52.40 & $(43-59) \quad 50.60$ & (43-80) 57.46 & $(45-61) 52.86$ & (14-81) 55.76 & $(42-57) \quad 50.93$ \\
\hline Bowman $(\mu \mathrm{m})$ & (9-19) 14.07 & (9-19) 15.37 & $(9-21) 14.7$ & (13-19) 15.23 & (8-18) 13.63 & $(11-18) 15.13$ \\
\hline Estroma $(\mu \mathrm{m})$ & (465-801) 589.76 & $(415-493) 444.43$ & (489-1024) 675.3 & $(413-530) 457.4$ & $(468-979) 673.96$ & $(411-515) 462.6$ \\
\hline $\begin{array}{l}\text { Descemet/ } \\
\text { Endotelio }(\mu \mathrm{m})\end{array}$ & (12-19) 15.16 & $(11-22) \quad 15.93$ & (11-33) 16.46 & 16.00 & $(11-28) 15.63$ & (11-19) 15.4 \\
\hline \multirow[t]{2}{*}{ Total $(\mu \mathrm{m})$} & $(550-882) 671.46$ & $(498-571) 526.33$ & (569-1141) 763.93 & (496-617) 541.16 & (545-1076) 758 & (491-598) 544.33 \\
\hline & \multicolumn{2}{|c|}{$p<0.0001$} & \multicolumn{2}{|c|}{$\mathrm{p}<0.0001$} & \multicolumn{2}{|c|}{$p<0.0001$} \\
\hline
\end{tabular}

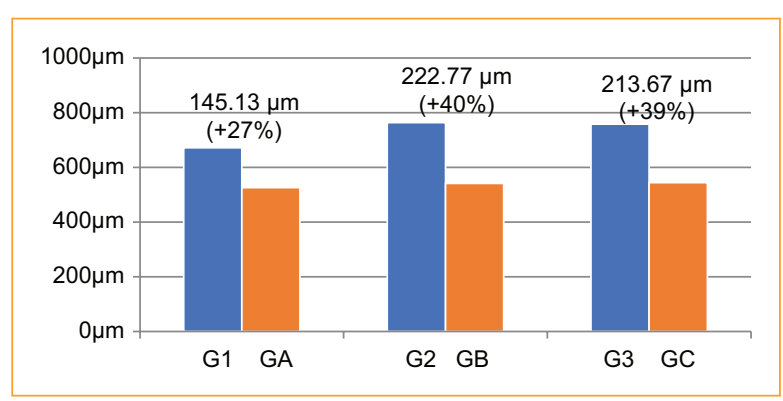

Figura 1. Grosor corneal. Grupos 1, 2 y 3 corresponden a los ojos operados de facoemulsificación. Grupos A, B y $C$ corresponden a los ojos contralaterales no operados. Se compara el aumento de grosor corneal entre los ojos operados y los no operados.

de los ojos operados con los ojos controles (Tabla 2). Se puede apreciar que la densidad total disminuye a expensas de la densidad estromal, de la membrana de Descemet y el endotelio. Se encuentra una disminución proporcional significativa de la densidad corneal de $3.33(-2.01 \%)$ en el grupo 1 , de $6.8(-4.13 \%)$ en el grupo 2 y de $8.55(-5.07 \%)$ en el grupo 3 de los pacientes operados; a diferencia del grosor corneal, aquí la edad no desempeña un factor protector (Fig. 3).

La reflectividad corneal fue similar en los ojos operados y en los ojos controles en las tres capas superficiales de la córnea, pero las capas de la membrana de Descemet y el endotelio de los grupos control se aprecian hiperreflécticas. En comparación con las córneas de los ojos operados, se encontraron las capas hiperreflécticas, hiporreflécticas y en un mínimo porcentaje (6.66\%) capas con características hiperreflécticas en algunos segmentos de la membrana de Descemet y el endotelio, esto cuando se supera un EPT $>8$ segundos (Tabla 3 ).

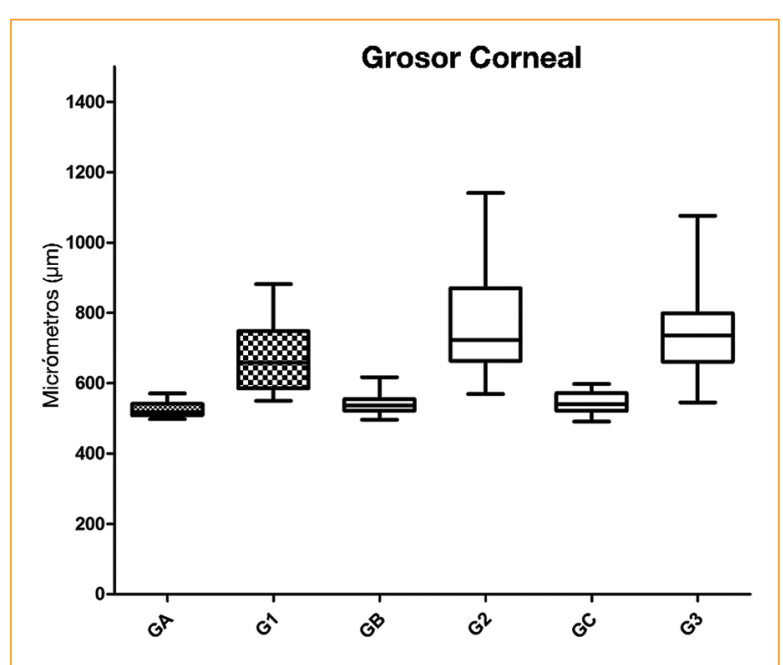

Figura 2. Grosor corneal. Grupos 1, 2 y 3 corresponden a los ojos operados de facoemulsificación. Grupos A, B y C corresponden a los ojos contralaterales no operados. Se compara el aumento de grosor corneal entre los ojos operados y los no operados. Existe un cambio significativo entre el grupo de 50 años y los grupos de 60 y 70 años.

Las características principales encontradas en las córneas operadas fueron edema estromal en el 100\%, hiperreflectividad en la membrana de Descemet y el endotelio en el $64 \%$ (Fig. 4), pliegues en la membrana de Descemet en el $47 \%$, y desprendimiento de la membrana de Descemet en el 5.55\% (Fig. 5 y Tabla 4).

En las pruebas de correlación se encontró que el aumento del grosor estromal es directamente proporcional al aumento del EPT (0.5963; $p<0.0001)$; sin embargo, la densidad estromal es inversamente proporcional $(-0.1908 ; p=0.0359)$ y la densidad normal de la membrana de Descemet y el endotelio es 
Tabla 2. Densidad corneal. Los grupos 1, 2 y 3 corresponden a los ojos operados de facoemulsificación, y los grupos $A, B$ y $C$ corresponden a los ojos contralaterales no operados. Se presenta el valor promedio de densidad en escala grises 256 píxeles 8-bit (G2 $\mathrm{b}$ ), y los valores que aparecen entre paréntesis corresponden a los máximos y mínimos

\begin{tabular}{|c|c|c|c|c|c|c|}
\hline & \multicolumn{2}{|c|}{ 50-59 años $(n=60)$} & \multicolumn{2}{|c|}{ 60-69 años (n = 60) } & \multicolumn{2}{|c|}{ 70-79 aи̃os ( $n=60$ ) } \\
\hline Grupos & $1(\mathrm{n}=30)$ & $A(n=30)$ & $2(\mathrm{n}=30)$ & $B(n=30)$ & $3(n=30)$ & $C(n=30)$ \\
\hline Epitelio (G28 b) & (96-255) 198.28 & (78-255) 181.16 & (109-255) 212.35 & (91- 255) 182.35 & (101-255) 207.03 & (100-255) 184.4 \\
\hline Bowman $\left(\mathrm{G} 2^{8} \mathrm{~b}\right)$ & (98-229) 169.83 & (101-221) 157.3 & $(90-228) 180.46$ & (125-241) 168.95 & (133-240) 191.45 & (127-228) 179.11 \\
\hline Estroma (G28 $\mathrm{b})$ & $(40-252) 148.16$ & $(68-251) 162.61$ & $(20-253) 143.15$ & $(68-251) 162.43$ & (38-243) 138.96 & (89-240) 166.73 \\
\hline $\begin{array}{l}\text { Descemet/ } \\
\text { endotelio ( } \mathrm{G} 2^{8} \mathrm{~b} \text { ) }\end{array}$ & (22-247) 109.2 & (34-245) 137.98 & $(25-231) 94.91$ & $(41-244) 143.41$ & (30-208) 97.8 & $(53-228) 141.48$ \\
\hline \multirow[t]{2}{*}{ Total $\left(G 2^{8} \mathrm{~b}\right)$} & $\begin{array}{c}(140.12-196.12) \\
156.37\end{array}$ & $\begin{array}{c}(144.5-172.5) \\
159.7\end{array}$ & $\begin{array}{c}(140.75-178.62) \\
157.61\end{array}$ & $\begin{array}{c}(148.87-177.5) \\
164.41\end{array}$ & $\begin{array}{c}(142.12-172.12) \\
160.05\end{array}$ & $\begin{array}{c}(160.12-176.75) \\
168.6\end{array}$ \\
\hline & \multicolumn{2}{|c|}{$p=0.0028$} & \multicolumn{2}{|c|}{$p=0.0011$} & \multicolumn{2}{|c|}{$p<0.0001$} \\
\hline
\end{tabular}

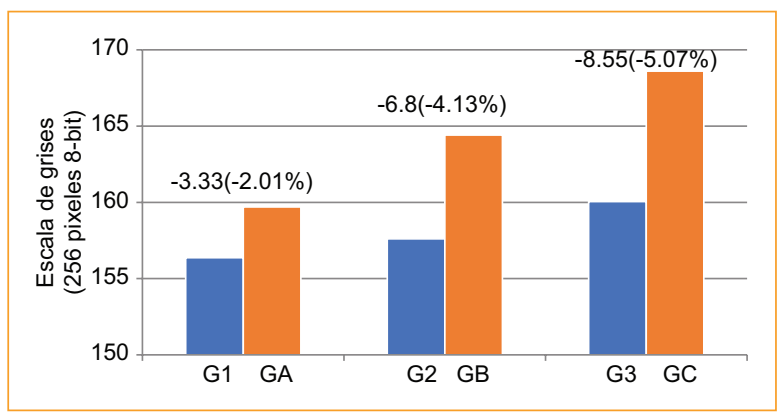

Figura 3. Densidad Corneal. Grupos 1, 2 y 3 corresponde a los ojos operados de facoemulsificación. Grupos $A, B$ y $C$ corresponden a los ojos contralaterales no operados. Se comparan la disminución de densidad corneal entre los ojos operados y los no operados, se representa en escala grises 256 pixeles 8 -bit $\left(G 2^{8} \mathrm{~b}\right)$, los valores que aparecen entre paréntesis corresponden a la disminución en porcentaje.

inversamente proporcional cuando supera los 8 segundos de EPT (-0.5957; $p<0.0001)$ (Figs. 6 y 7 ).

\section{Discusión}

El uso del ultrasonido durante la cirugía de catarata modifica la arquitectura corneal de forma proporcional, ya que cuantos más segundos de tiempo efectivo de facoemulsificación se utilicen, más significativos serán los cambios anatómicos. Sharma, et al. ${ }^{9}$ encontraron que existe edema corneal en todos los pacientes operados de facoemulsificación independientemente de los segundos utilizados; en este estudio se encontró el mismo edema en todas las córneas de los ojos operados, pero también se observó que este edema no es similar en todos los grupos de edad, así como tampoco la densidad corneal a partir de 8 segundos de EPT.

Durante el análisis corneal mediante las imágenes de OCT-SD en los ojos no operados se observó que la máxima reflectividad se encuentra en el epitelio y la mínima en el estroma, debido a que el estroma es la capa de la córnea que se encuentra más hidratada. En los ojos operados con facoemulsificación, la reflectividad endotelial disminuye al mínimo conforme disminuye la densidad estromal, observándose que los ojos operados del grupo de 50 años presentaban la mayor reflectividad endotelial, debido a que cuanto menor es la edad, mayor densidad celular endotelial puede tener el paciente; sin embargo, no hubo prácticamente diferencia entre los grupos de 60 y 70 años.

Los desprendimientos de la membrana de Descemet tras la facoemulsificación se han clasificado en tres tipos: simple, que solo afecta la zona de la incisión; simétrico, que afecta la zona de incisión y la zona contralateral; y completo, que afecta el centro de la córnea. En el presente estudio se identificaron cinco casos de desprendimiento de membrana de Descemet: uno en el grupo de 50 años, otro en el grupo de 60 años y tres en el grupo de 70 años. La edad desempeña un papel importante, siendo más probable que a mayor edad se desprenda más fácilmente la membrana de Descemet durante la facoemulsificación.

No existen muchos estudios que hayan utilizado la OCT-SD para describir las características corneales tras la cirugía de catarata, ya que la mayoría de las veces se da por hecho que los cambios anatómicos se 
Tabla 3. Reflectividad corneal. Los grupos 1, 2 y 3 corresponden a los ojos operados de facoemulsificación, y los grupos $A, B$ y C corresponden a los ojos contralaterales no operados. Se representan los resultados en hiperreflécticos e hiporreflécticos dependiendo de la reflectividad observada por capa en las imágenes de tomografía de coherencia óptica de dominio espectral. Se presentan el número de ojos según la reflectividad encontrada en cada capa corneal, y los valores que aparecen entre paréntesis corresponden al porcentaje encontrado en cada grupo

\begin{tabular}{|c|c|c|c|c|c|c|}
\hline \multirow[b]{2}{*}{ Grupos } & \multicolumn{2}{|c|}{$50-59$ años $(n=60)$} & \multicolumn{2}{|c|}{ 60-69 años (n = 60) } & \multicolumn{2}{|c|}{ 70-79 años (n = 60) } \\
\hline & $1(n=30)$ & $A(n=30)$ & $2(n=30)$ & $B(n=30)$ & $3(n=30)$ & $C(n=30)$ \\
\hline Epitelio & \multicolumn{6}{|c|}{ Hiperrefléctico 180 (100\%) } \\
\hline Bowman & \multicolumn{6}{|c|}{ Hiperrefléctico $180(100 \%)$} \\
\hline Estroma & \multicolumn{6}{|c|}{ Hiporrefléctico 180 (100\%) } \\
\hline $\begin{array}{l}\text { Descemet/endotelio } G \\
\text { A, B y C } \\
(n=90)\end{array}$ & \multicolumn{6}{|c|}{ Hiperrefléctico 90 (100\%) } \\
\hline $\begin{array}{l}\text { Descemet/endotelio G } 1 \\
(n=30)\end{array}$ & \multicolumn{2}{|c|}{ Hiperrefléctico 18 (60\%) } & \multicolumn{2}{|c|}{$\begin{array}{l}\text { Hiperrefléctico/hiporrefléctico } \\
6(20 \%)\end{array}$} & \multicolumn{2}{|c|}{$\begin{array}{l}\text { Hiporrefléctico } \\
6(20 \%)\end{array}$} \\
\hline $\begin{array}{l}\text { Descemet/endotelio G } 2 \\
(n=30)\end{array}$ & \multicolumn{2}{|c|}{ Hiperrefléctico 17 (56.66\%) } & \multicolumn{2}{|c|}{$\begin{array}{l}\text { Hiperrefléctico/hiporrefléctico } \\
22(6.66 \%)\end{array}$} & \multicolumn{2}{|c|}{$\begin{array}{l}\text { Hiporrefléctico } \\
11(33.66 \%)\end{array}$} \\
\hline $\begin{array}{l}\text { Descemet/endotelio G } 3 \\
(\mathrm{n}=30)\end{array}$ & \multicolumn{2}{|c|}{ Hiperrefléctico 12 (40\%) } & \multicolumn{2}{|c|}{$\begin{array}{l}\text { Hiperrefléctico/hiporrefléctico } \\
2(6.66 \%)\end{array}$} & \multicolumn{2}{|c|}{$\begin{array}{l}\text { Hiporrefléctico } \\
16(53.33 \%)\end{array}$} \\
\hline
\end{tabular}

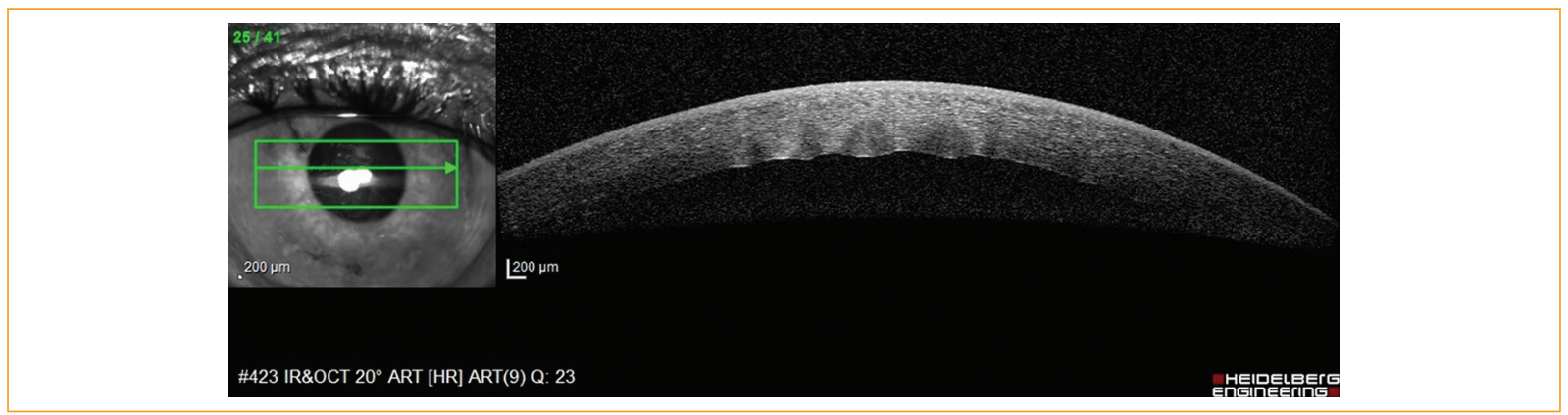

Figura 4. Pliegues en membrana de Descemet. Se observó por medio de OCT-SD pliegues en la membrana de Descemet en el $47 \%$ de los paciente operados de facoemulsificación.

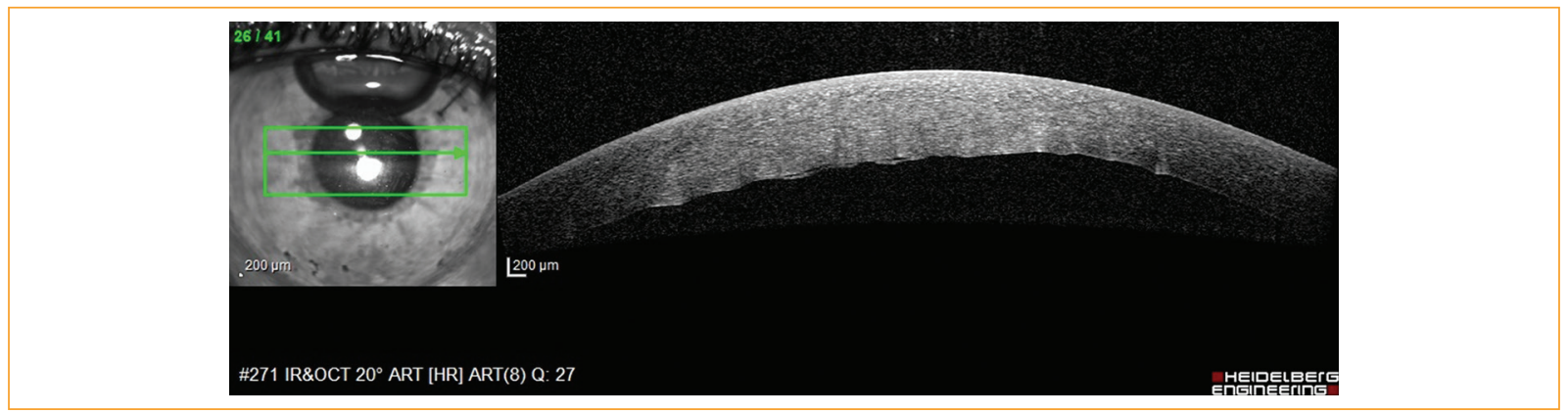

Figura 5. Edema estromal. Se observó por medio de OCT-SD edema estromal en el $100 \%$ de los paciente operados de facoemulsificación. 
Tabla 4. Características corneales tras la facoemulsificación. Los grupos 1, 2 y 3 corresponden a los ojos operados de facoemulsificación. Se representa cómo el edema estromal estuvo presente en el 100\% de los casos, la hiperreflectividad endotelial disminuye conforme aumenta la edad, los pliegues que se formaron en la membrana de Descemet no demostraron diferencia significativa entre grupos de edad y finalmente el desprendimiento de membrana de Descemet se encontró en tres pacientes del grupo $3(10 \%)$, por lo que se sugiere que la edad puede ser un factor determinante para que esta se desprenda más fácilmente

\begin{tabular}{|l|c|c|c|c|}
\hline & Grupo 1 (50-59 años) & Grupo 2 (60-69 años) \\
\hline Edema estromal & $100 \%$ & $100 \%$ \\
\hline Hiperreflectividad endotelial & $80 \%$ & $64 \%$ & $100 \%$ \\
\hline Pliegues en membrana de Descemet & $39 \%$ & $53 \%$ & $47 \%$ \\
\hline Desprendimiento de membrana de Descemet & $3 \%$ & $3 \%$ & $10 \%$
\end{tabular}

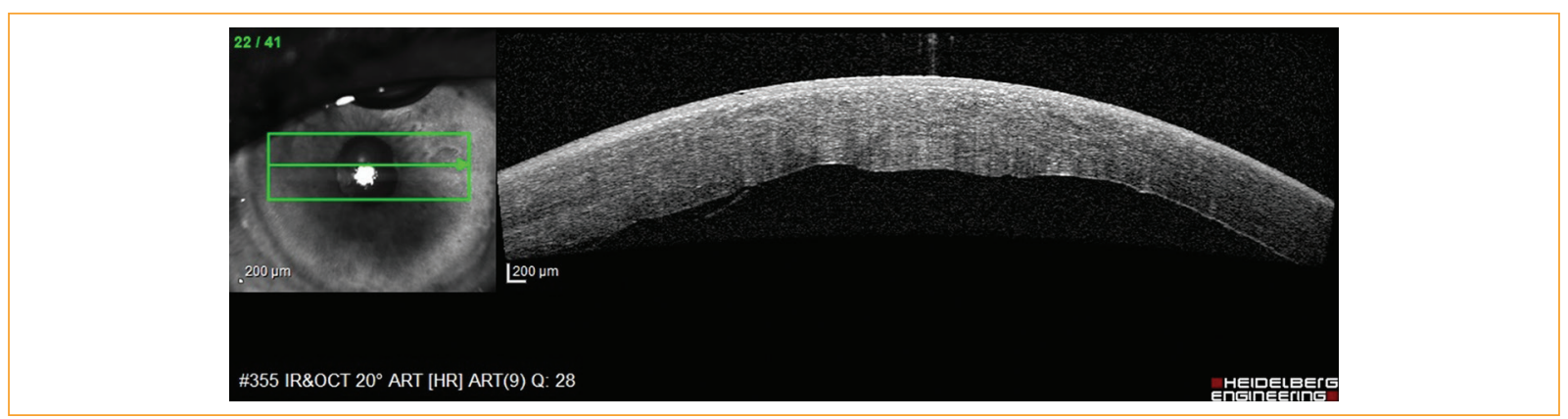

Figura 6. Desprendimiento de la membrana de Descemet. Se observó por medio de OCT-SD desprendimiento de la membrana de Descemet en 5 pacientes operados de facoemulsificación, en donde 3 pacientes corresponden al grupo etario de 70-79 años.
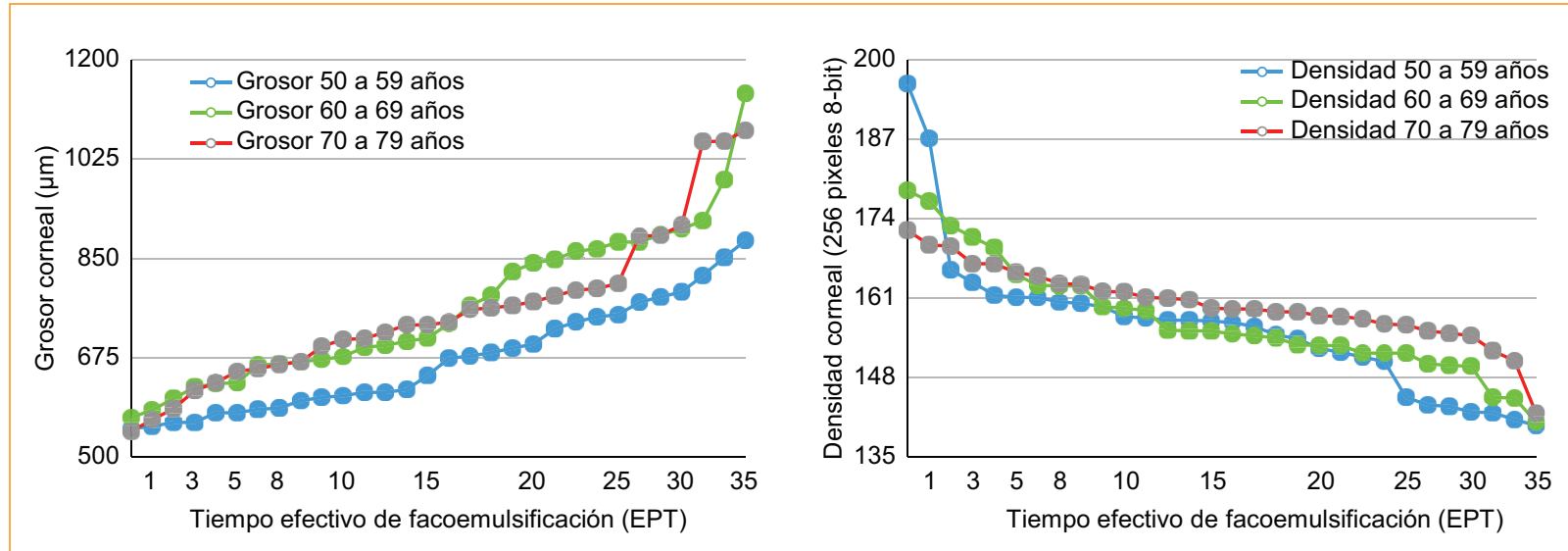

Figura 7. Comparación del grosor con la densidad corneal. En las pruebas de correlación se encontró que el aumento del grosor corneal es directamente proporcional al aumento del tiempo efectivo de facoemulsificación (EPT) (0.5963; $p<0.0001)$; sin embargo, la densidad estromal es inversamente proporcional al EPT $(-0.5957 ; p<0.0001)$.

resolverán con el uso correcto de los fármacos indicados en el posquirúrgico inmediato; sin embargo, sugerimos que, además de la biomicroscopía con lámpara de hendidura, es de utilidad utilizar las herramientas tecnológicas con que contamos actualmente para establecer un diagnóstico y un tratamiento oportuno ante cualquier complicación, así como explicarle el pronóstico visual al paciente. 


\section{Conclusiones}

Los cambios corneales posquirúrgicos no son inocuos y resulta fundamental seguir definiendo las características en las diferentes capas por métodos objetivos, como la OCT-SD, debido a que algunas alteraciones requieren un tratamiento oportuno. El aumento del grosor corneal es inversamente proporcional a la densidad, aunque la edad puede ser un factor predictor dependiendo del uso del ultrasonido durante la facoemulsificación. La reflectividad corneal presenta cambios significativos a partir de un EPT > 8 segundos, siendo esto un factor a considerar durante la cirugía de catarata. Finalmente, la facoemulsificación produce múltiples cambios corneales observables por OCT-SD, a pesar de que en la lámpara de hendidura en ocasiones no se perciban.

\section{Financiamiento}

Los autores declaran que el estudio no recibió ningún tipo de financiación o aportación económica.

\section{Conflicto de intereses}

Los autores declaran no tener ningún conflicto de intereses.

\section{Responsabilidades éticas}

Protección de personas y animales. Los autores declaran que para esta investigación no se han realizado experimentos en seres humanos ni en animales.

Confidencialidad de los datos. Los autores declaran que en este artículo no aparecen datos de pacientes.

Derecho a la privacidad y consentimiento informado. Los autores han obtenido el consentimiento informado de los pacientes y/o sujetos referidos en el artículo. Este documento obra en poder del autor de correspondencia.

\section{Bibliografía}

1. Testoni PA. Optical coherence tomography. Scientific World J. 2007;7: 87-108.

2. Sakata LM, Wong TTL, Wong H-T, Kumar RS, Htoon HM, Aung HT, et al. Comparison of Visante and slit-lamp anterior segment optical coherence tomography in imaging the anterior chamber angle. Eye. 2010;224:578-87.

3. Xia Y, Liu X, Luo L, Zeng Y, Cai X, Zeng M, et al. Early changes in clear cornea incision after phacoemulsification: an anterior segment optical coherence tomography study. Acta Ophthalmol (Oxf). 2010;87:764-8.

4. Calladine D, Packard R. Clear corneal incision architecture in the immediate postoperative period evaluated using optical coherence tomography. J Cataract Refract Surg. 2007;33:1429-35.

5. Choi CY, Youm DJ, Kim MJ, Tchah H. Changes in central corneal thickness of preserved corneas over time measured using anterior segment optical coherence tomography. Cornea. 2009;28:536-40.

6. Yi DH, Dana MR. Corneal edema after cataract surgery: Incidence and etiology. Semin Ophthalmol. 2002;17:110-4.

7. Benai CA, Tsao JZ, Afshari NA. Descemet membrane detachment during cataract surgery: etiology and management. Curr Opin Ophthalmol. 2017;28:35-41.

8. Ozcelik ND, Eltutar K, Bilgin B. Toxic anterior segment syndrome after uncomplicated cataract surgery. Eur J Ophthalmol. 2010;20:106-14.

9. Sharma N, Gupta S, Maharana P, Shanmugam P, Nagpal R, Vajpayee RB, et al. Anterior segment optical coherence tomography-guided management algorithm for Descemet membrane detachment after intraocular surgery. Cornea. 2015;34:1170-4.

10. Jacob S, Agarwal A, Chaudhry P, Narasimhan S, Chaudhry VN. A new clinico-tomographic classification and management algorithm for Descemet's membrane detachment. Cont Lens Anterior Eye. 2015;38:327-33.

11. Rubowitz A, Assia El, Rosner M, Topaz M. Antioxidant protection against corneal damage by free radicals during phacoemulsification. Invest Ophthalmol Vis Sci. 2003:44:1866-70.

12. Narayanan R, Gaster RN, Kenney MC. Pseudophakic corneal edema: a review of mechanisms and treatments. Cornea. 2006;25:993-1004.

13. Bodh SA, Kumar V, Raina UK, Ghosh B, Thakar M. Inflammatory glaucoma. Oman J Ophthalmol. 2011;4:3-9.

14. Wang T. Analysis and treatment of detachment of corneal posterior elastic layer during cataract phacoemulsification. Int $\mathrm{J}$ Ophthalmol. 2014;8:1511-2.

15. Guo P, Pan Y, Zhang Y, Tighe S, Zhu Y, Li M, et al. Study on the classification of Descemet membrane detachment after cataract surgery with AS-OCT. Int J Med Sci. 2018;15:1092-7.

16. Li H, Leung CKS, Wong L, Cheung CYL, Pang CP, Weinreb RN, et al. Comparative study of central corneal thickness measurement with slitlamp optical coherence tomography and Visante optical coherence tomography. Ophthalmology. 2008;115:796-801.

17. Baikoff G, Lutun E, Ferraz C, Wei J. Static and dynamic analysis of the anterior segment with optical coherence tomography. J Cataract Refract Surg. 2004;30:1843-50.

18. Dada T, Sihota R, Gadia R, Aggarwal A, Mandal S, Gupta V. Comparison of anterior segment optical coherence tomography and ultrasound biomicroscopy for assessment of the anterior segment. J Cataract Refract Surg. 2007;33:837-40. 